\title{
Notes on the Dipole-Moment Derivatives of $\mathrm{PF}_{3}$
}

Recently Levin and Adams $(I)$ calculated the dipole-moment derivatives with respect to the symmetry coordinates $\partial \mathbf{y} / \partial S_{j}$ for $\mathrm{PF}_{3}$ from the absolute infrared intensities, and indicated a preferred set of derivatives through comparison with $\mathrm{CNDO} / 2$ calculations. However, the published $L^{-1}$ matrix elements for the $A_{1}$-symmetry species are not consistent with $L \tilde{L}=G,{ }^{1}$ and as a consequence the $\partial \mathbf{u} / \partial S_{j}$ values in that symmetry block are in error.

We have redetermined the normal coordinate vectors and the dipole-moment derivatives for the $A_{1}$-species, where we used the same geometry, symmetry coordinates and force field as Levin and Adams (1). The results are listed in Table I, which may be compared with the Tables II and III of (1).

The remaining sets of sign combinations of the $\partial \mathbf{y} / \partial Q_{i}$ 's would only reverse the signs of the $\partial \mu_{z} / \partial S_{j^{-}}$ values and not their magnitudes. Levin and Adams stated, that also in the $E$-species, where a correction for the compensating rotation of the molecule is necessary, two sets of sign combinations are enough to

${ }^{1}$ In fact, the error occurs in the $L_{12}$-element.

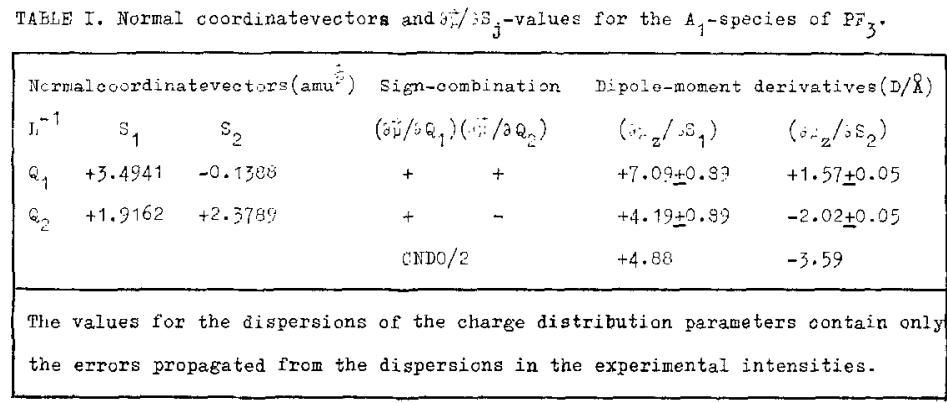

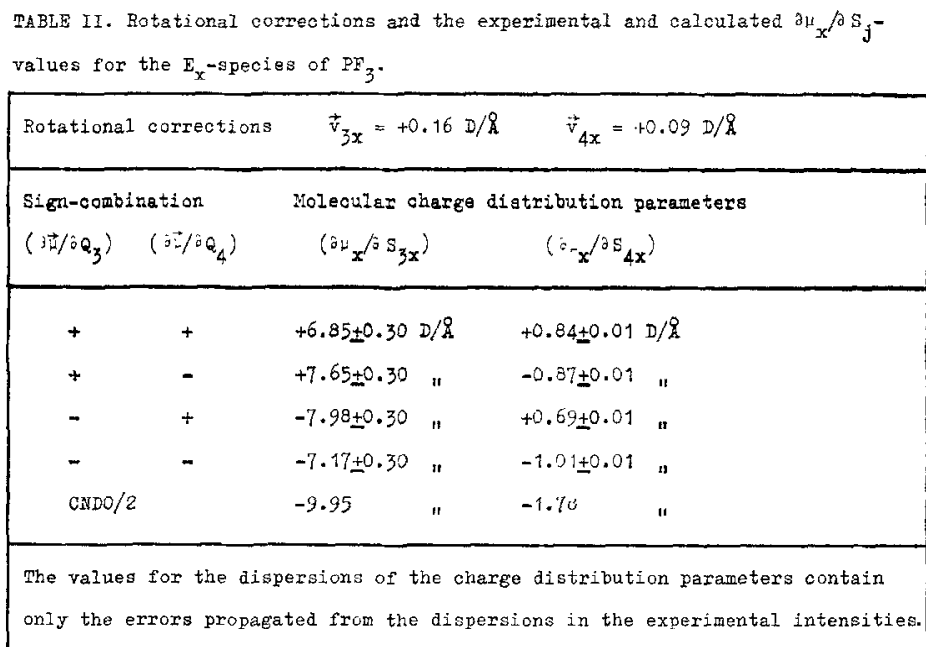


indicate all possible magnitudes of the $\partial \mu_{x} / \partial S_{j}$-values. However, in our opinion, in this case not two but four sets of sign combinations are needed.

In Table II we present the rotational corrections required to correct $\partial \mu_{x} / \partial S_{j}$ for the rotation of the permanent moment and the corrected experimental and calculated dipole-moment derivatives. Comparison with the $\mathrm{CNDO} / 2$ results led to the following values for the $\partial u / \partial S_{j}$-quantities.

$$
\begin{aligned}
& A_{1} \text {-species: } \quad \partial \mu_{z} / \partial S_{1}=+4.19 \pm 0.89 \mathrm{D} / \AA, \quad \partial \mu_{z} / \partial S_{2}=-2.02 \pm 0.05 \mathrm{D} / \AA .
\end{aligned}
$$

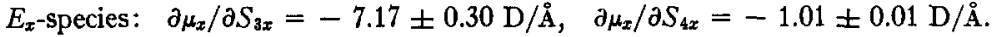

\section{REFERENCE}

1. I. W. Levin and O. W. Adaus, J. Mol. Spectrose., 39, 380 (1971).

Analytical Chemisiry Laboratory,

A. J. van Straten and W, M. A. Suit

State Universily of Utrecht,

Croesestraat $77 A$, Utrecht, The Netherlands

Received March 6, 1974 